\title{
Selection of suitable reference genes for reverse transcription-quantitative polymerase chain reaction analysis of neuronal cells differentiated from bone mesenchymal stem cells
}

\author{
YU-XI HE* , YAN ZHANG* , QIWEI YANG, CHENGUANG WANG and GUANFANG SU \\ Department of Ophthalmology, Second Hospital, Jilin University, Changchun, Jilin 130000, P.R. China
}

Received July 2, 2014; Accepted March 16, 2015

DOI: $10.3892 / \mathrm{mmr} .2015 .3671$

\begin{abstract}
Reverse transcription-quantitative polymerase chain reaction (RT-qPCR) is a technique widely used for the quantification of mRNA transcription, It is well recognized that the reference genes used in RT-qPCR require appropriate validation to ensure that gene expression is unaffected by experimental conditions. The differentiation of bone mesenchymal stem cells (BMSCs) into neurons is important in the treatment of nerve injury. In gene expression analysis of the differentiation of BMSCs into neuronal cells by, the commonly used reference genes for RNA analysis are often selected without any preliminary evaluation of their suitability. The present study aimed to evaluate the mRNA expression levels of 11 putative reference genes, including ACTB, ARBP, B2M, CYCA, GAPDH, GUSB, HPRT, PPIA, RPL13A, TBP and PGK1, in order to select the most suitable reference genes for RT-qPCR of the differentiation of neuronal cells by BMSCs. The mRNA expression levels of the 11 putative reference genes were examined using RT-qPCR in rat BMSCs differentiated into neuronal cells. Normal BMSCs and three types of rat BMSCs, which were chemically induced to differentiate into neurons using neurotrophic cytokines and co-culture with retinal cells. The geNorm, NormFinder and BestKeeper software programs were used to select the most suitable reference genes. The results of the analyses using the three software programs demonstrated that RPL13A was the most stable among all the groups, while ACTB was the least stable. The combination of CYCA and PPIA reference genes contributed the most to increasing stability. The suitability of selected reference genes requires previous pre-selection in every investigation. Based on the three software programs, RPL13A, and
\end{abstract}

Correspondence to: Dr Guanfang Su, Department of Ophthalmology, Second Hospital, Jilin University, 218 Ziqiang Street, Changchun, Jilin 130000, P.R. China

E-mail:sugf2012@163.com

*Contributed equally

Key words: bone mesenchymal stem cell, differentiation, reference gene, quantitative polymerase chain reaction, normalization the combination of CYCA and PPIA were identified as the most suitable reference genes for RT-qPCR in neuronal cells differentiated from BMSCs.

\section{Introduction}

Reverse transcription-quantitative polymerase chain reaction (RT-qPCR) is widely used to quantify RNA expression levels (1). RT-qPCR is highly sensitive, allowing the quantification of rare transcripts (2). However, the accuracy of this technique may be affected at multiple stages throughout the experimental process and by several factors, including the quantity of the samples analyzed, the quality of the RNA, the efficiencies of RT and the PCR itself $(3,4)$. The stability of the reference gene is important for an appropriate normalization standard $(5,6)$. An ideal reference gene requires it not to be regulated or affected in any sample under different experimental treatment conditions (7). However, no single universal and completely constant reference gene has been reported (8). Accumulating evidence has indicated that the expression levels of widely used reference genes vary significantly in different independent investigations $(7,9,10)$. Several housekeeping genes, commonly used as reference genes, can be dynamically expressed in response to treatment (11). Therefore, the identification and evaluation of the expression stabilities of reference genes is required to obtain accurate profiling of gene expression levels.

The plasticity of BMSCs offers potential in the progress of investigation into regeneration in neural tissues, and offers a foundation for the application of stem cells in diseases of the nervous system (12). It has been reported that BMSCs can be induced to differentiate into neuron-like cells in vitro and in vivo, which can be induced by various factors and differentiated into several specific neuronal phenotypes $(13,14)$. Woodbury et al, was the first to induce rat and human BMSCs to differentiate into neuron-like and glial-like cells in vitro (13). Our previous study demonstrated that the retinal tissue offered an inductive microenvironment to promote stem cell differentiation into neural-like cells with morphological characteristics of nerve cells, and promoted the expression of nestin and nuclear factor (15). Several types of cytokines have also been used to induce BMSCs to differentiate into neuron-like cells in vitro. These cells can promote functional recovery and neural protection following spinal cord 
injury (12). During development, BMSCs undergo complex differentiation procedures and phenotypic changes. To realize the potential of these cells, it is necessary to understand the processes, which govern their differentiation (16).

In the present study, the expression stabilities of 11 commonly used reference genes, ACTB, ARBP, B2M, CYCA, GAPDH, GUSB, HPRT, PPIA, RPL13A, TBP and PGK1, were investigated. The commonly used statistical algorithms, geNorm (17), NormFinder (18) and BestKeeper (19) were used to evaluate their expression stabilities.

The results of the present study may assist in determining the optimal number of reference genes required for reliable normalization of gene expression data.

\section{Materials and methods}

Isolation, culture and identification of BMSCs. Neonatal (8-day-old; 3 rats, 15-25 g) Wistar rats, provided by the Animal Center of Jilin University (Jilin, China), were housed together at $19-29^{\circ} \mathrm{C}$ with a $12 \mathrm{~h}$ light/dark cycle, and were placed in $75 \%$ alcohol (Beijing Sinopharm Chemical Reagent Co., Ltd., Beijing, China) for sterilization for $5 \mathrm{~min}$. The BMSCs were harvested from the bone marrow of the femur of neonatal female Wistar rats under $10 \%$ chloral hydrate anesthetic (Tianjin Huadong Chemical Reagent Co., Ltd., Tianjin, China; 1 ml; intraperitoneal injection), by inserting a needle into the bone shaft and repeatedly flushing it with $5 \mathrm{ml}$ Dulbecco's modified Eagle's medium (DMEM)/F12 medium (GE Healthcare Bio-Sciences, Pittsburgh, PA, USA). The rinsed solution was transferred slowly into a centrifuge tube, which contained equal quantities of $1.073 \mathrm{~g} / 1$ percol separation liquid (Sigma-Aldrich, St. Louis, MO, USA), and centrifuged (Legend Micro 17R; Thermo Fisher Scientific, Waltham, MA, USA) at $350 \mathrm{x}$ g for $20 \mathrm{~min}$ at room temperature. The middle monolayer was then collected and washed twice with phosphate-buffered saline (PBS; Wuhan Boster Biological Technology, Ltd., Wuhan, China). The cells were maintained in a humidified incubator (Sanyo, Osaka, Japan) in an atmosphere of $5 \% \mathrm{CO}_{2}$ at $37^{\circ} \mathrm{C}$ with DMEM/F12 medium and $5 \%$ fetal bovine serum (FBS; Gibco Life Technologies, Carslbad, CA, USA). After $24 \mathrm{~h}$, the non-adherent cells were removed by medium replacement, and the medium was replaced every 3 days. The cells were passaged when they reached $90 \%$ confluence to maintain exponential growth. Third-generation BMSCs were used for the subsequent experiments.

Following three passages, the expression levels of the CD90, CD71, CD29 and CD45 cell surface markers (BD Biosciences, Franklin Lakes, NJ, USA) were detected by flow cytometry (Epics XL; Beckman Coulter, Brea, CA, USA).

Differentiation of BMSCs. The third passage BMSCs were seeded into six-well plates at a density of 20,000 cells $/ \mathrm{cm}^{2}$, and were cultured at $\sim 4 \times 10^{4}$ cells $/ \mathrm{cm}^{2}$ in growth medium until confluent, prior to being divided into four groups. In the blank group, BMSCs contained no inducer. In the chemical group, the BMSCs were maintained in $\beta$-mercaptoethanol (BME; Beijing Dingguo Company, Beijing, China) in six-well plates at $1 \mathrm{mmol} / 1$ for $24 \mathrm{~h}$ pre-induction at $37^{\circ} \mathrm{C}$, to induce neuronal differentiation, the cells were then transferred into serum-free medium containing $5 \mathrm{mmol} / \mathrm{l} \mathrm{BME} \mathrm{(13).} \mathrm{In}$ the cytokine group, the BMSCs were maintained in basic fibroblast growth factor (Peprotech company), induced at $37^{\circ} \mathrm{C}$ for $24 \mathrm{~h}$ at $20 \mathrm{ng} / \mathrm{ml}$, and the medium was replaced with serum-free medium containing $10 \mathrm{ng} / \mathrm{ml}$ brain-derived neurotrophic factor (Peprotech company) (20). In the co-culture group, the eyeballs of six 3-day-old Wistar rats were removed under sterile conditions. The eyeball was cut from the limbus, the lens and vitreous were removed under a microscope (CKX41; Olympus, Tokyo, Japan) and the retina was separated. For digestion, $1 \mathrm{ml} 0.125 \%$ trypsin/EDTA (GE Healthcare Life Sciences) was added to the retinal tissue and incubated at $37^{\circ} \mathrm{C}$ for $10 \mathrm{~min}$ until the retinal tissue became chyle. This was followed by the addition of $2 \mathrm{ml} \mathrm{DMEM} / \mathrm{F} 12$ medium containing 10\% FBS to terminate the digestion. The cells were then centrifuged at $60 \mathrm{x} g$ for $5 \mathrm{~min}$ at room temperature, and the cells were resuspended and maintained in a humidified incubator in $5 \% \mathrm{CO}_{2}$ at $37^{\circ} \mathrm{C}$. The medium was replaced every 3 days and the medium was collected, The differentiation medium was mixed with the collected medium and fresh medium (2:3). The differentiation medium was used for the third passage BMSCs.

Immunohistochemistry was performed to detect the expression levels of the nestin, neuron-specific enolase (NSE) and neurofilament (NF) neuronal markers (Abcam, Cambridge, MA, USA) in the induced groups. The frozen cell sections were hydrated with distilled water for $30 \mathrm{~min}$, then $0.2 \%$ Triton X-100 (Beijing Dingguo Company) was added for $20 \mathrm{~min}$ prior to washing (with 0.2 M PBS, 3 times, 5 min each time). The cells were blocked with methanol and $30 \%$ hydrogen peroxide (50:1) (both from Beijing Sinopharm Chemical Reagent Co., Ltd.) at room temperature for $30 \mathrm{~min}$, then $5 \%$ bovine serum albumin (Sigma-Aldrich) blocking buffer was added at room temperature for $20 \mathrm{~min}$. Excess buffer was then removed. The cells were incubated with the primary antibody at $4^{\circ} \mathrm{C}$ overnight, were washed 3 times with $0.2 \mathrm{M}$ PBS (5 min each time), then were incubated with the secondary antibody at room temperature for $1 \mathrm{~h}$. Washing was then conducted (with 0.2 M PBS, 3 times, 5 min each time). Streptavidin-biotin complex (Wuhan Boster Biological Technology, Ltd.) was added at room temperature for $20 \mathrm{~min}$, then the cells were washed (with $0.2 \mathrm{M}$ PBS, 3 times, 5 min each time). The DAB kit (Wuhan Boster Biological Technology, Ltd.) was then used in accordance with the manufacturer's instructions, allowing color to develop for $30 \mathrm{~min}$ prior to washing with water. Hematoxylin (Nanchang Yvlu Chemical Reagent Co., Ltd., Jiangxi, China) staining was conducted for $5 \mathrm{~min}$, the cells were dehydrated, cleared using xylene and fixed with $40 \mu \mathrm{l}$ neutral gum (Nanchang Yvlu Chemical Reagent Co., Ltd.), then imaged under the microscope, the positive results appearing brown.

RNA extraction and cDNA synthesis. Total RNA was isolated from the independent biological replicates, each group containing two samples, and were isolated using TRIzol reagent (Invitrogen Life Technologies), according to the manufacturer's instructions. The quantity and purity of the total RNA extracted was estimated by monitoring the absorbance at the ratio 260/280 using a NanoDrop 2000 spectrophotometer (Thermo Fisher Scientific, Waltham, MA, USA). The 260/280 ratio did not vary significantly among the groups. The integrity of the $28 \mathrm{~S}$ and $18 \mathrm{~S}$ ribosomal 
Table I. Reference genes for gene expression normalization in induced bone mesenchymal stem cells.

\begin{tabular}{|c|c|c|c|c|c|}
\hline Gene & Accession no. & $\begin{array}{l}\text { Amplicon } \\
\text { length (bp) }\end{array}$ & Sequence $\left(5^{\prime}-3^{\prime}\right)$ & E & $\mathrm{R}^{2}$ \\
\hline TBP & NM 001004198.1 & 111 & $\begin{array}{l}\text { Sense TAATCCCAAGCGGTTTGCTG } \\
\text { Antisense TTCTTCACTCTTGGCTCCTGTG }\end{array}$ & 2.12 & 0.998 \\
\hline B2M & NM 012512.1 & 114 & $\begin{array}{l}\text { Sense CGAGACCGATGTATATGCTTGC } \\
\text { Antisense GTCCAGATGATTCAGAGCTCCA }\end{array}$ & 1.94 & 0.986 \\
\hline HPRT & NM 012583.2 & 152 & $\begin{array}{l}\text { Sense TCCCAGCGTCGTGATTAGTGA } \\
\text { Antisense CCTTCATGACATCTCGAGCAAG }\end{array}$ & 2.08 & 0.975 \\
\hline RPL13A & NM 173340.2 & 145 & $\begin{array}{l}\text { Sense TCTCCGAAAGCGGATGAACAC } \\
\text { Antisense CAACACCTTGAGGCGTTCCA }\end{array}$ & 1.92 & 0.991 \\
\hline ARPB & NM 022402.1 & 165 & $\begin{array}{l}\text { Sense CCCTTCTCCTTCGGGCTGAT } \\
\text { Antisense TGAGGCAACAGTCGGGTAGC }\end{array}$ & 1.90 & 0.985 \\
\hline ACTB & NM 031144.2 & 86 & $\begin{array}{l}\text { Sense CTGACTGACTACCTCATGAAGATCCT } \\
\text { Antisense CTTAATGTCACGCACGATTTCC }\end{array}$ & 1.83 & 0.946 \\
\hline PGK1 & NM 053291.3 & 113 & $\begin{array}{l}\text { Sense GCAGATTGTTTGGAACGGTCC } \\
\text { Antisense TAGTGATGCAGCCCCTAGACGT }\end{array}$ & 2.05 & 0.943 \\
\hline PPIA & NM 017101.1 & 135 & $\begin{array}{l}\text { Sense CCAAACACAAATGGTTCCCAGT } \\
\text { Antisense ATTCCTGGACCCAAAACGCT }\end{array}$ & 1.88 & 0.990 \\
\hline GUSB & NM 017015.2 & 101 & $\begin{array}{l}\text { Sense CCTTTCTACTTCCAAGGCGTCA } \\
\text { Antisense CAACGGAGGAGGTTGAAATCC }\end{array}$ & 1.74 & 0.993 \\
\hline CYCA & NM 017101.1 & 126 & $\begin{array}{l}\text { Sense TATCTGCACTGCAAGACTGAGTG } \\
\text { Antisense CTTCTTGCTGGTCTTGCCATTCC }\end{array}$ & 1.98 & 0.986 \\
\hline GAPDH & NM 17008.4 & 223 & $\begin{array}{l}\text { Sense GGCATTGCTCTCAATGACAA } \\
\text { Antisense TGTGAGGGAGATGCTCAGTG }\end{array}$ & 1.90 & 0.981 \\
\hline
\end{tabular}

Each sample contained three biological replicates. E, reaction efficiency; $\mathrm{R}^{2}$, Pearson's coefficient of determination.

RNA was confirmed using $1 \%$ agarose gel electrophoresis (Sigma-Aldrich) with $500 \mathrm{ng}$ of the total RNA. The ethidium-bromide (Beijing Dingguo Company) stained gels were exposed to ultraviolet light (FS-312; Shanghai Fusheng Institute of Biotechnology, Shanghai, China), to determine the quality of the RNA.

For cDNA synthesis, $1 \mu \mathrm{g}$ total RNA from each pooled sample was reverse transcribed into cDNA using a PrimeScript RT Reagent kit (Takara Bio, Inc., Dalian, China), according to the manufacturer's instructions, in a $20 \mu \mathrm{l}$ reaction volume. All the cDNA samples were stored at $-20^{\circ} \mathrm{C}$ until qPCR analyses.

Quantification of $m R N A$. The primers of the ACTB, ARBP, B2M, CYCA, GAPDH, GUSB, HPRT, PPIA, RPL13A, TBP and PGK1 reference genes were synthesized by Sangon Company (Shanghai, China; Table I). The primers were designed using the following criteria: A temperature between $58-62^{\circ} \mathrm{C}$ and optimal temperature of $60^{\circ} \mathrm{C}$; a PCR product size between 75 and 200 base pairs, a length of between 18 and 24 nucleotides and optimal length of 20 nucleotides; and a GC content between 40 and $60 \%$. The primers were assessed using BLAST analysis to verify the specificity and selective amplification of the target gene, as primers can obtain amplification of cDNA and not genomic DNA.
The mRNA expression levels were analyzed by RT-qPCR. Equal quantities of DNA-free RNA from each sample were used. The PCRs were performed using SGExcelFastSYBR mix, containing ROX (SK2954B; Sangon Company). The PCR mix in each well contained $5 \mu 1$ of $2 \mathrm{X}$ SGExcelFastSYBR

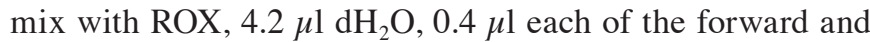
reverse primers $(10 \mathrm{pmol} / \mu \mathrm{l})$ and $0.4 \mu \mathrm{l}$ single strand cDNA $(50 \mathrm{ng} / \mu \mathrm{l})$ in a final reaction volume of $10 \mu \mathrm{l}$. Amplification was performed on a LightCycler 480 (Roche Diagnostics, Basel, Switzerland) using the following program: $72^{\circ} \mathrm{C}$ for $5 \mathrm{~min}$; $94^{\circ} \mathrm{C}$ for $3 \mathrm{~min}$, stage $3: 94^{\circ} \mathrm{C}$ for $20 \mathrm{sec}, 57^{\circ} \mathrm{C}$ for $20 \mathrm{sec}$ and $72^{\circ} \mathrm{C}$ for $20 \mathrm{sec}$ for 40 cycles; and $72^{\circ} \mathrm{C}$ for $3 \mathrm{~min}$. To minimize variation in the $\mathrm{RT}$ reaction, all the RNA samples from a single experimental setup were reverse transcribed simultaneously. The qPCR products were subjected to $3 \%$ agarose gel electrophoresis.

To estimate the efficiencies of amplification, a standard curve was generated for each primer pair, based on four points of a serial 2-fold dilution of cDNA (40.0, 4.0, 0.4 and $0.04 \mathrm{ng}$ ). The amplification efficiencies and the correlation coefficient $\left(\mathrm{R}^{2}\right)$ values were calculated using the slope of the calibration curve, using the following equation: $E=2^{-1 / \text { slope }}$.

The threshold cycle values were collected for export using the PCR system software (LightCycler 480 software, version 1.5.0) for further analysis. 
Statistical analysis. To analyze and confirm the results, the geNorm (17), NormFinder (18) and BestKeeper (19) applications were used. These bioinformatics packages calculate stability values. The genes were then ranked in order of stability, according to these obtained gene expression stability values.

GeNorm-based analysis of candidate reference genes. GeNorm software is designed to determine an accurate selection for a set of genes, which exhibit minimal variation across different biological conditions (17). It uses the average pair-wise variation between a particular gene and all other reference genes, and calculates the optimal number of genes necessary for normalization. This analysis provides a ranking of the assessed genes based on their stability measure (M), determining the most stable reference genes or a combination of multiple stable genes for normalization.

The $\mathrm{M}$ value denotes the mean pair-wise variation for a specific gene compared with other assessed genes, The gene with the highest $M$ value is then excluded from the analysis, and recalculated in order to select the two most stable genes. The reference genes are ranked according to their $\mathrm{M}$ value, between the lowest and the highest. Lower M values represent higher expression stabilities.

NormFinder-based analysis of candidate reference genes. NormFinder computes the expression stability values using an analysis of variance-based model (18). NormFinder is a mathematical approach for identifying the optimal gene to use as reference genes among a set of candidates. It uses all candidate genes and provides a ranking order based on the estimated intragroup stability of the candidate genes, based on an estimate of the variation in each subgroup, and intergroup stability, based on the combination of all subgroups. The two results are then combined, to produce a stability value for each gene investigated. The program ranks genes based on a stability value, with the lowest value indicating the most stably expressed gene.

BestKeeper analysis of candidate reference genes. BestKeeper analysis is based on the threshold cycle $(\mathrm{Ct})$ values and is presented as the standard deviation (SD) and coefficient of correlation $\left(\mathrm{R}^{2}\right)(19)$. The genes with an $\mathrm{SD}>1.00$ are considered unreliable as a stable reference gene. The remaining genes are ranked according to their $r$ values, higher $r$ values indicating higher stability. A novel feature of BestKeeper is the calculation of the intrinsic variance of expression for a single sample, which detects outliers. Feng et al previously described that the lowest ranking genes in geNorm and NormFinder were the same (21), however BestKeeper was not included in their analysis.

\section{Results}

Culture and identification of BMSCs. The majority of the cells grew adherently following inoculation for $24 \mathrm{~h}$, were spindly with adequate refraction (Fig. 1), were mixed with other types of cells, and certain cells were suspended in the culture medium. The non-adherent cells were removed by replacing the medium. The cells reached a confluence of up to $90 \%$ after
Table II. Analysis of the expression stabilities of the 11 reference genes using NormFinder software.

\begin{tabular}{lcc}
\hline Reference gene & Stability value & Rank \\
\hline RPL13A & 0.158 & 1 \\
PPIA & 0.205 & 2 \\
HPRT & 0.230 & 3 \\
CYCA & 0.280 & 4 \\
ARBP & 0.319 & 5 \\
TBP & 0.559 & 6 \\
GAPDH & 0.618 & 7 \\
PGK1 & 0.625 & 8 \\
B2M & 0.687 & 9 \\
GUSB & 0.784 & 10 \\
ACTB & 1.186 & 11 \\
\hline
\end{tabular}

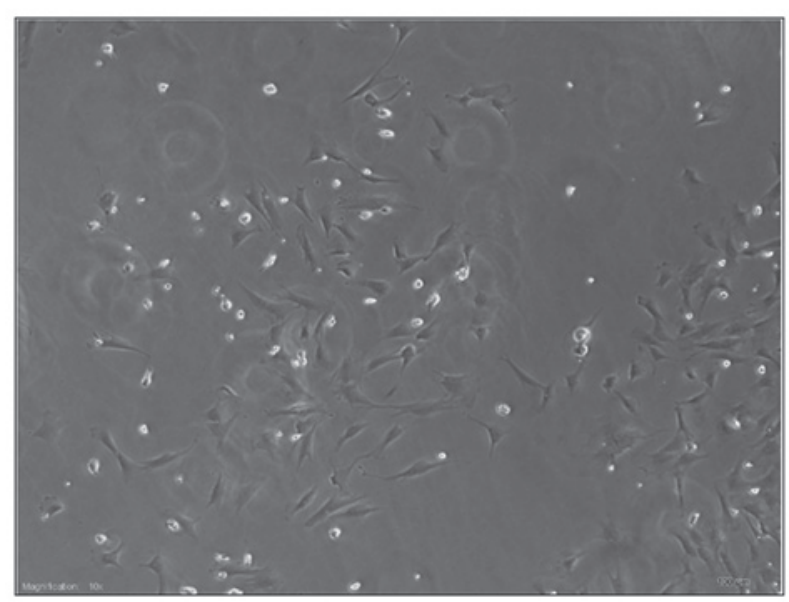

Figure 1. Primary bone mesenchymal stem cells. The BMSCs grew adherently and were spindly with adequate refraction. (CKX41 microscope; magnification, $\mathrm{x} 100)$.

5-7 days, following which the growth rate accelerated. The cells were passaged once after 3-5 days and, at the third passage, the cells exhibited a spindle-shaped fibroblastic morphology. Fluorescent cell sorting at passage three demonstrated that the cells were negative for CD45, and positive for CD90, CD71 and CD29 (Fig. 2).

Differentiation of BMSCs. Following the addition of inducers, the cell bodies became increasingly spherical and refractive, exhibiting a typical neuronal appearance. The processes continued to grow, exhibiting primary and secondary branches, growth cone-like terminal expansions and putative filopodial extensions. To further characterize the neuronal differentiation, the cells were stained for the neuronal markers, nestin, NSE and NF (Fig. 3).

Amplification performance of the primers. The qPCR amplification product was detected using 3\% agarose gel electrophoresis with the expected size and no primer dimmers, (Fig. 4A). For relative quantification, a standard curve was generated in each individual run by serially diluting the pool 

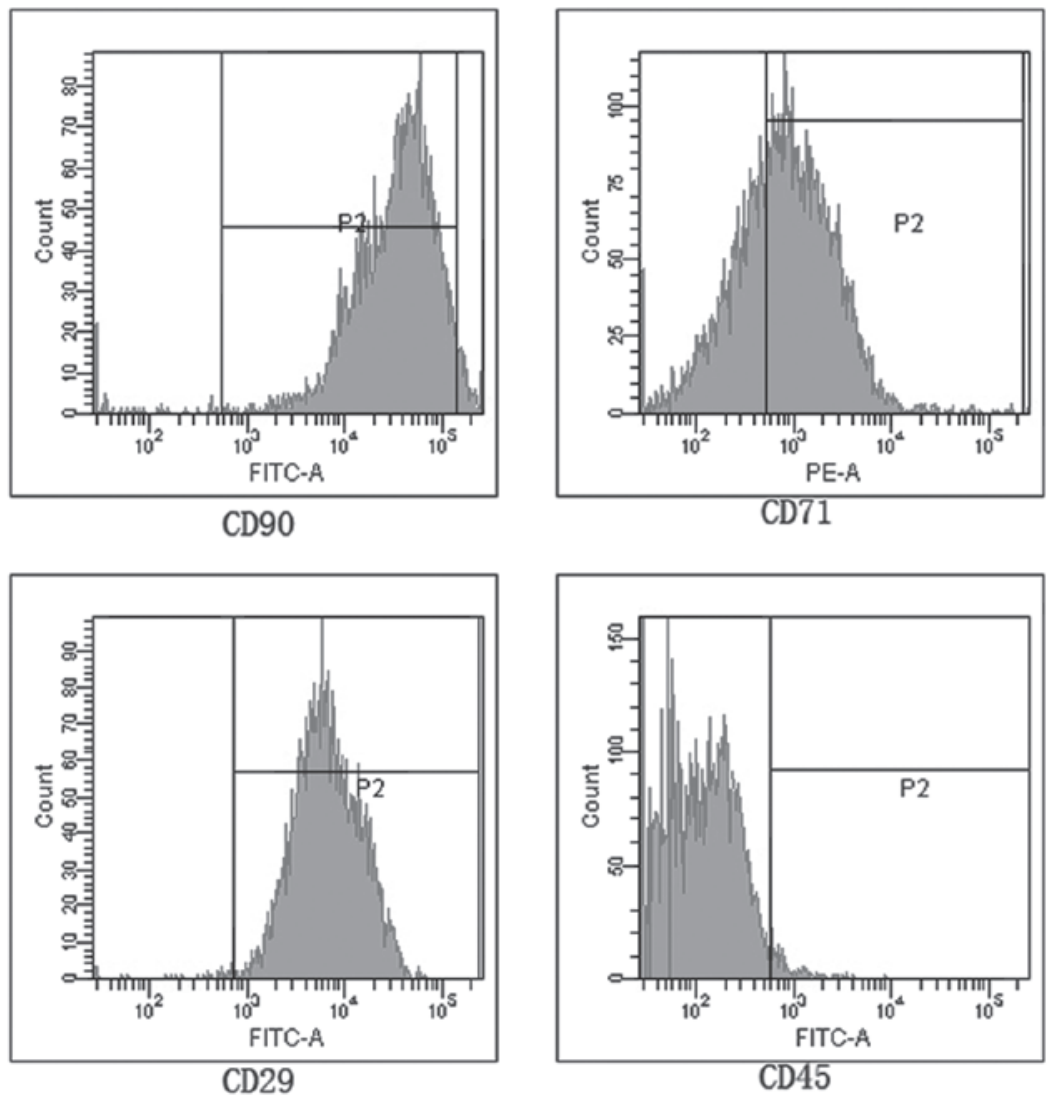

Figure 2. Bone mesenchymal stem cell surface markers are negative for CD45, and positive for CD90, CD71 and CD29. Results were produced using flow cytometry. FITC, fluorescein isothiocyanate.
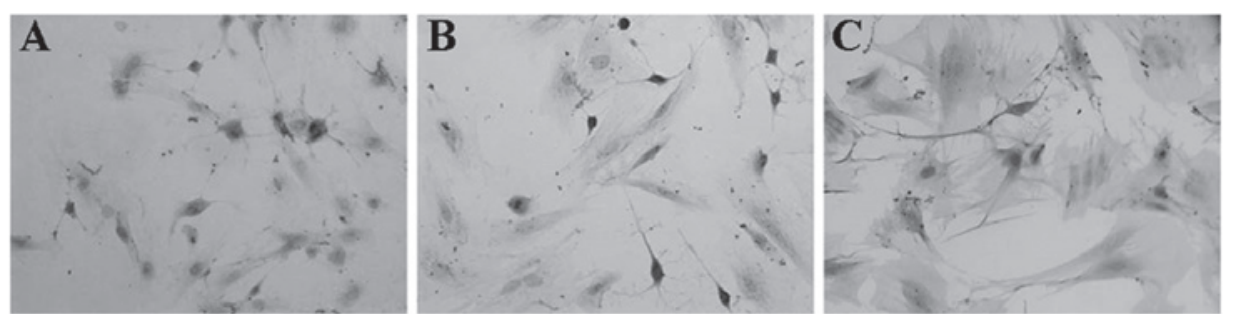

Figure 3. Histochemical staining with hematoxylin of three types of BMSC-derived neuron cells. (A) Positive staining for nestin was observed following induction of BMSCs by $\beta$-mercaptoethanol (magnification, x200). (B) Positive staining for neurofilament following induction of BMSCs by brain-derived neurotrophic factor (magnification, x200). (C) Positive staining for neuron-specific enolase following induction of BMSCs by co-culture medium (magnification, $\mathrm{x} 200)$ BMSC, bone mesenchymal stem cell.

of cDNA samples (40.0, 4.0, 0.4 and $0.04 \mathrm{ng}$ ) with high expression values. One single peak was obtained in each amplification reaction on analyzing the melting curves, which confirmed the specific amplification of The primers (Fig. 4B).

For all 11 genes, the amplification efficiencies were between 1.74 and 2.11 , and the correlation coefficients $\left(\mathrm{R}_{2}\right)$ were $>0.94$.

Expression profile of the reference genes. Each sample was analyzed in duplicate, and the average $\mathrm{Ct}$ value for each sample and the coefficient of variation (CV) for each groups was obtained. The $\mathrm{CV}$, expressed as percentage and calculated as the $\mathrm{SD} /$ mean $\mathrm{Ct}$, was used to compare the degree of variation among the 11 reference genes. The $\mathrm{Ct}$ values for each of the 11 candidate reference genes ranged between 13 and 25 cycles.
Figure 5 shows the mean $\mathrm{Ct}$ values of each gene in the four groups of treated cells.

The reference genes exhibited $\mathrm{Ct}$ values between 13.63 for GAPDH and 24.53 for TBP. These genes exhibited different $\mathrm{Ct}$ values, GAPDH exhibited the lowest $\mathrm{Ct}$ values, between 13.63 and 15.94, while TBP exhibited the highest $\mathrm{Ct}$ values, between 22.93 and 24.53. ACTB exhibited the highest variation in levels of RNA expression ( $\mathrm{SD}=2.59)$. The lowest variation $\mathrm{i}$ levels of RNA expression was observed for TBP $(\mathrm{SD}=0.58)$, followed by CYCA $(\mathrm{SD}=0.60)$

Expression stability of candidate reference genes using GeNorm. The geNorm analysis software was used to select the optimal reference genes. The parameters, which were considered to quantify reference gene stability were the $M$ value and 
A

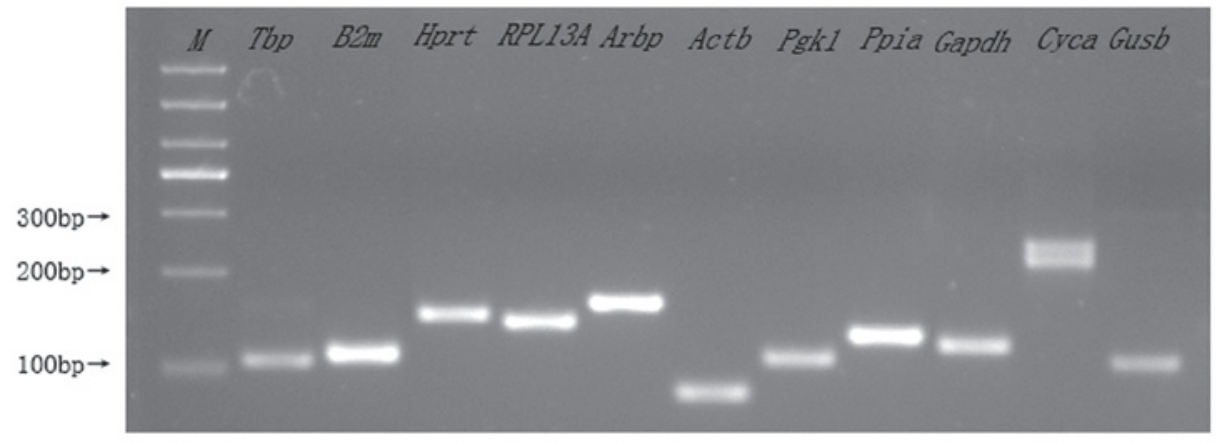

B
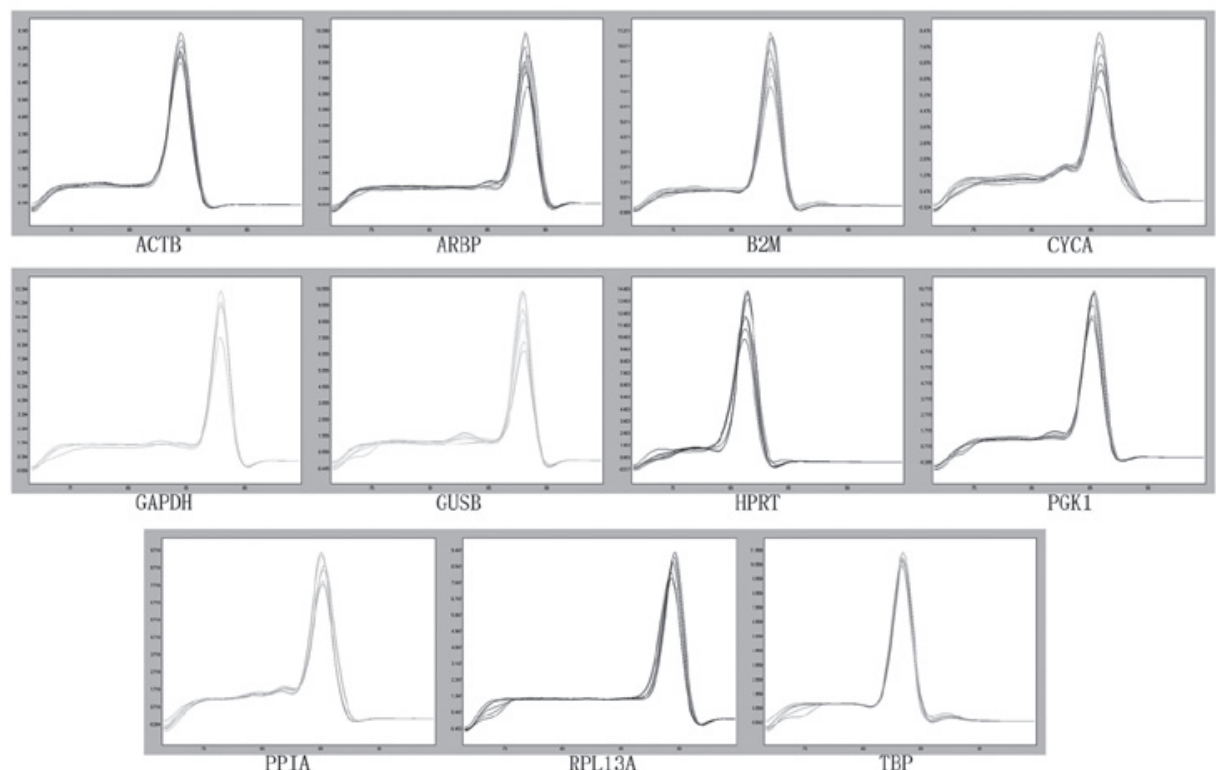

Figure 4. Specificity of primers and amplicon lengths. (A) Amplification of fragments using 3\% agarose gel electrophoresis, the amplification product of primers showed expected size and no primer dimmers. (B) Melting curves for each of the reference genes, one single peak was obtained in each amplification reaction. $\mathrm{M}$, marker.

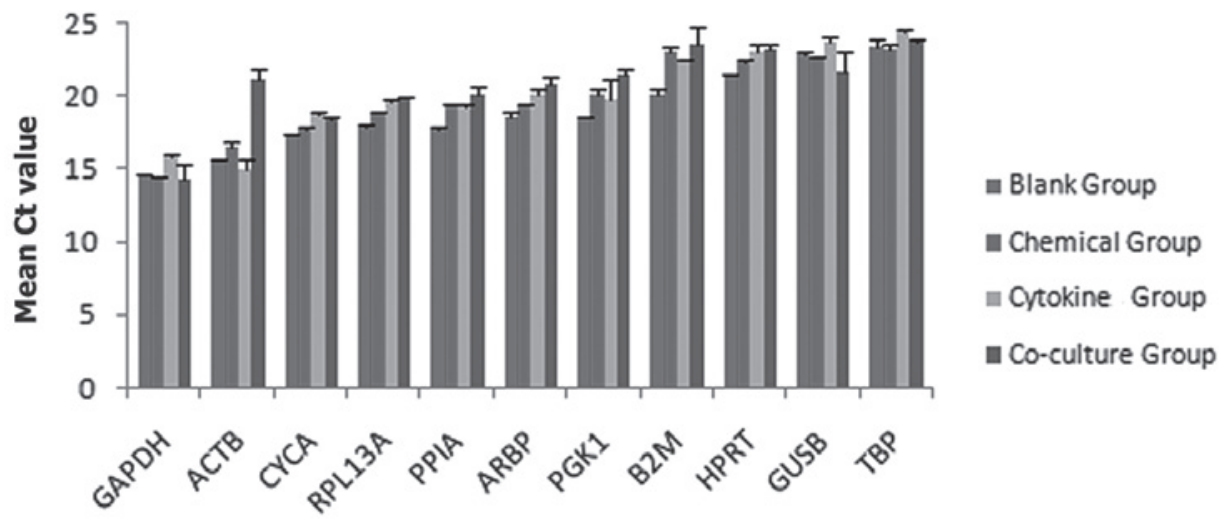

Figure 5. Mean Ct values of the candidate reference genes across the experimental groups. Blank group, BMSCs without any inducer derived mRNA samples; chemical group, BMSCs with chemical inducer-derived mRNA samples; cytokine group, BMSCs with cytokine inducer-derived mRNA samples; co-culture group, BMSCs with co-culture medium inducer-derived mRNA samples. Data are expressed as the mean \pm standard deviation. BMC, bone mesenchymal stem cells. Ct, threshold cycle.

pair-wise variation. The software eliminates genes with the highest M-value, and repeats the process until there only two genes remain. An $\mathrm{M}$ value $<1.5$ indicates that no genes are considered unreliable as a stable reference gene. Among the 11 genes, no M value were $>1.5$. RPL13A and HPRT were the most stable genes, exhibiting the lowest $\mathrm{M}$ values, and ACTB was the least stable gene, exhibiting the highest $\mathrm{M}$ value (Fig. 6A).

To determine the optimal number of required reference genes for each group, the $\mathrm{Vn} / \mathrm{n}+1$ was evaluated using geNorm. For all the $\mathrm{Vn} / \mathrm{n}+1$ values, including $\mathrm{V} 2 / 3$, indicating the number of further reference genes to add on the normalization 
Table III. Descriptive statistics of the 11 reference genes based on threshold cycle values, analyzed using BestKeeper software.

\begin{tabular}{lrrrrrrrrrr}
\hline Value & GAPDH & CYCA & RPL13A & PPIA & ARBP & PGK1 & B2M & HPRT & GUSB & TBP \\
\hline Geo mean Ct & 14.80 & 18.06 & 19.05 & 19.16 & 19.76 & 19.93 & 22.21 & 22.51 & 22.67 & 23.66 \\
Ar mean Ct & 14.82 & 18.06 & 19.07 & 19.18 & 19.78 & 19.97 & 22.25 & 22.52 & 22.69 & 23.67 \\
Min Ct & 13.63 & 17.25 & 17.77 & 17.78 & 18.49 & 18.51 & 19.88 & 21.35 & 20.58 & 22.93 \\
Max Ct & 15.94 & 18.91 & 19.99 & 20.45 & 21.20 & 21.67 & 24.36 & 23.43 & 23.97 & 24.53 \\
SD of Ct & 0.56 & 0.52 & 0.70 & 0.69 & 0.69 & 1.06 & 1.08 & 0.61 & 0.60 & 0.45 \\
CV (\%) & 3.80 & 2.86 & 3.69 & 3.61 & 3.50 & 5.28 & 4.86 & 2.70 & 2.62 & 1.88 \\
Min (-fold) & -2.25 & -1.75 & -2.44 & -2.61 & -2.40 & -2.68 & -5.02 & -2.23 & -4.24 & -1.66 \\
Max(-fold) & 2.20 & 1.81 & 1.92 & 2.44 & 2.72 & 3.34 & 4.45 & 1.90 & 2.47 & 1.82 \\
SD ( \pm -fold) & 1.48 & 1.43 & 1.63 & 1.62 & 1.62 & 2.08 & 2.12 & 1.52 & 1.51 & 1.36 \\
CC (r) & 0.344 & 0.944 & 0.968 & 0.899 & 0.881 & 0.741 & 0.845 & 0.918 & -0.086 & 0.588
\end{tabular}

$\mathrm{n}=80 . \mathrm{Ct}$, cycle threshold; geo, geometric; ar, arithmeticmean; min, minimum; max; maximum; SD, standard deviation; CV, coefficient of variation based on $\mathrm{Ct}$; -fold, fold-change in $\mathrm{CV}$; CC, pairwise correlation coefficient. $\mathrm{SD}$ values $>1.00$ were eliminated from further analysis.

A

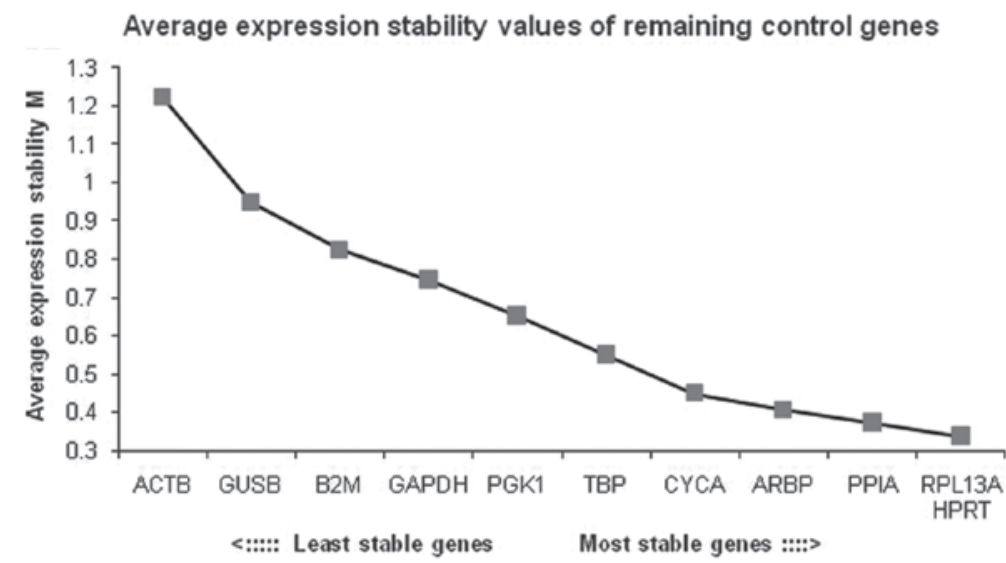

B

Determination of the optimal number of control genes for normalization

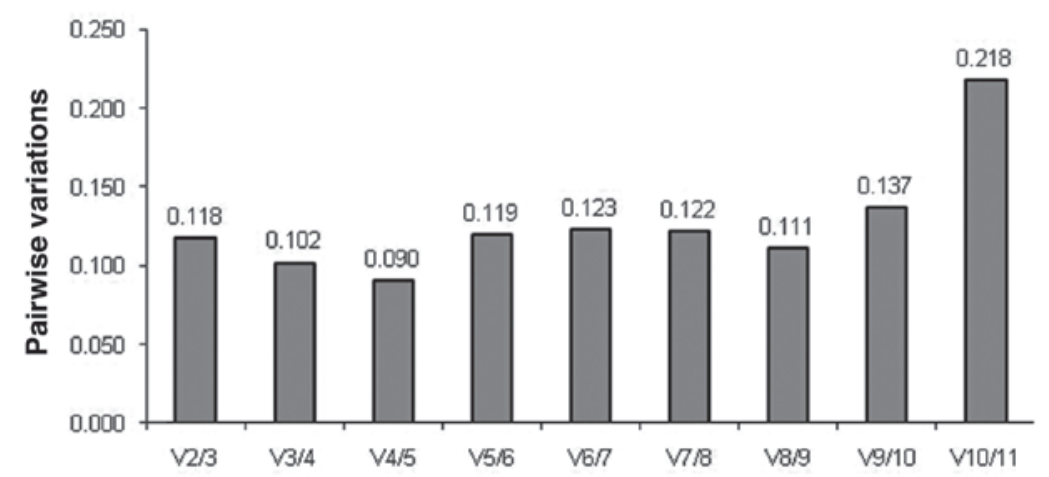

Figure 6. Stability values of the reference genes analyzed using geNorm sofware. (A) Expression stability measures (M) of reference genes analyzed. The $\mathrm{x}$-axis from left to right indicates the ranking of the genes according to their stability; lower $\mathrm{M}$ values indicate higher stabilities. (B) Determination of the suitable number of reference genes for normalization. The software calculates the normalization factor from at least two genes, at which the $\mathrm{V}$ value defines the pair-wise variation between two sequential normalization factors.

factor, a threshold of 0.15 was designed as the cut-off value for pair-wise variation. The inclusion of the third reference gene was not necessary (Fig. 6B).
NormFinder analysis. NormFinder was used to confirm the most stably expressed gene. As shown in Table II, a low stability value represents a low expression variance. The results 
Table IV. Ranking of reference genes using geNorm, NormFinder and BestKeeper analyses.

\begin{tabular}{cccc}
\hline Rank & geNorm & NormFinder & BestKeeper \\
\hline 1 & RPL13A/HPRT & RPL13A & RPL13A \\
2 & PPIA & CYCA & \\
3 & PIPA & HPRT & HPRT \\
4 & ARBP & CYCA, & PPIA \\
5 & CYCA & ARBP & ARBP \\
6 & TBP & TBP & TBP \\
7 & PGK1 & GAPDH & GAPDH \\
8 & GAPDH & PGK1 & GUSB \\
9 & B2M & B2M & \\
10 & GUSB & GUSB & \\
11 & ACTB & ACTB & \\
\hline
\end{tabular}

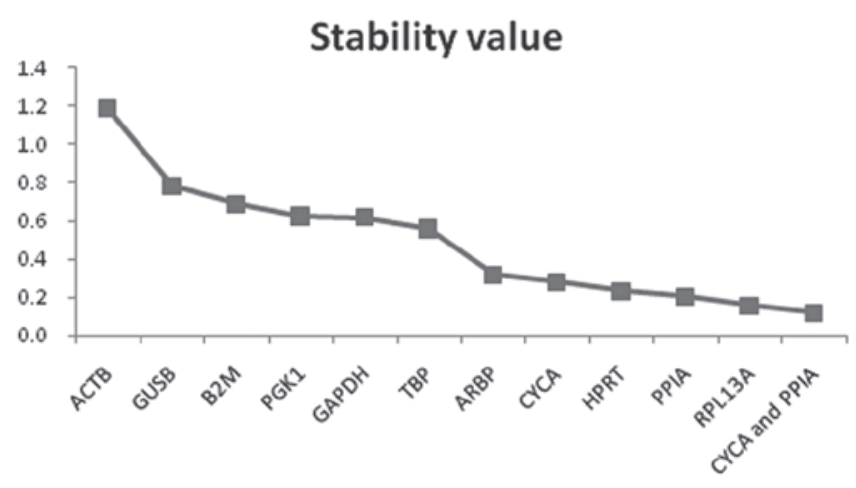

Figure 7. Stability values of the reference genes, calculated using NormFinder software. A lower stability value indicates higher stability.

demonstrated that RPL13A was the most stablly expressed gene, with a stability value of 0.158 , and ACTB was the least stably expressed gene, with a stability value of 1.186 . These results were consistent with those of the geNorm analysis. The most suitable combination of genes was CYCA and PPIA, with a combined stability value of 0.119 , and their stability value was lower than RPL13A, therefore, this combination was confirmed as the most stable genes, providing a higher level of stability compared with using only one (Fig. 7).

BestKeeper analysis. BestKeeper was also used to identify the most stably expressed genes, by comparing the $r$ values and SD. The number of candidate reference genes was limited by this software tool, therefore, 10 of the candidate reference genes were analyzed. As geNorm and NormFinder identified ACTB with the most variance, ACTB was eliminated. The results revealed that the $\mathrm{SD}$ value of $\mathrm{B} 2 \mathrm{M}$ and $\mathrm{PGK} 1$ were $>1.00$ and were, therefore, considered unacceptable and eliminated (Fig. 8A). Among the remaining genes, according $r$ values of the candidate reference genes, the higher stability values implied increased stability, of which RPL13A exhibited the highest stability (Fig. 8B). The descriptive statistics of the reference genes based on their $\mathrm{Ct}$ values, analyzed using BestKeeper, are shown in Table III.
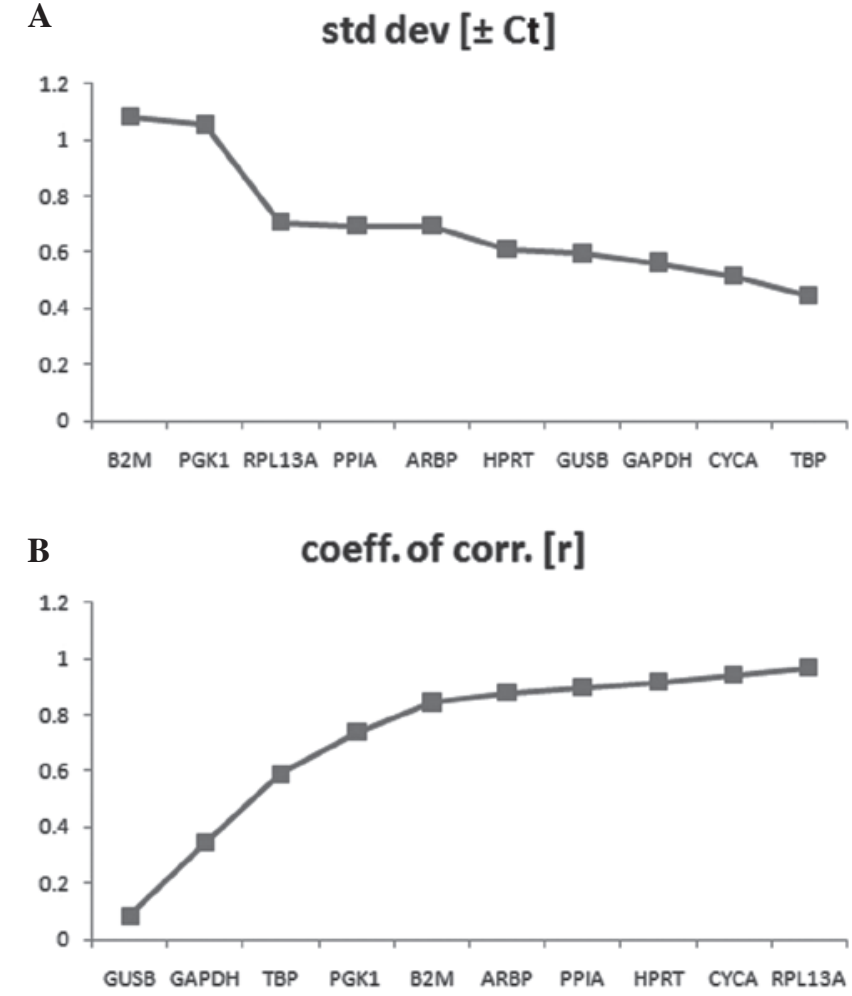

Figure 8. Stability values of the reference genes, evaluated using BestKeeper software. (A) Std dev values of the reference genes, any values $>1.00$ were eliminated from further analysis. (B) $r$ values of reference genes, high stability values indicate higher stability. Coeff. of corr, correlation of coefficient; std dev, standard deviation; $\mathrm{Ct}$, cycle threshold.

Ranking of the reference genes, using the results of the geNorm, NormFinder and BestKeeper analyses, are shown in Table IV.

\section{Discussion}

BMSCs are a type of stem cell, which are different from hematopoietic stem cells in bone marrow. They are a type of early mesodermal cell with multiple differentiation potentials. As the availability of pluripotent retinal stem cells is not limited (22), and are not rejected by the host immune system in either allogeneic or xenogenic host species recipients (23), BMSCs have become an area of interest. Several studies have demonstrated that BMSCs differentiate into chondrocytes, fibroblasts, adipocytes, cardiomyocytes and skeletal muscle cells in vitro (24-26). The differentiation of BMSCs into neuronal cells has been a focus of investigations. Studies have reported that they can be induced to differentiate into neuron-like cells in vitro and in vivo $(13,15,27)$. In vivo investigations have demonstrated that BMSCs can differentiate into neuron-like cells, and the neurogenesis, maturation and migration of the cells were the same as those observed in neural stem cells. The understanding of these cells and their differentiation potentials is important for their use to achieve desired functions in vivo. To evaluate the differentiation of the cells, determination of the mRNA levels of specific genes is necessary.

At present, qPCR is the most fundamental, accurate and commonly used method to investigate mRNA levels Due to its 
low cost and ease of operation, qPCR is consistently used to assess the expression levels of specific genes. As a crucial step requires that the RT-qPCR data is accurately normalized by use of appropriate reference genes, as the use of non-validated reference genes can lead to erroneous results, which are biologically meaningless (28). However, there are currently no universally applicable reference genes. The same reference genes in different species, and different reference genes in the same species, can have markedly different expression levels under diverse experimental conditions $(7,29)$. For example, during rat MSC differentiation into osteocyte, adipocyte and chondrocyte lineages, the GUSB and B2M reference genes were among the least stable genes in the MSC and adipocyte lineages, but were the most stable in the chondrocyte and osteocyte lineages (30).

To the best of our knowledge, there are no previous reports regarding the validation of reference genes for gene expression assays of rat BMSCs during neuronal differentiation. In the present study, the expression stabilities of 11 commonly accepted candidate reference genes was analyzed. The public programs, geNorm, NormFinder and BestKeeper, were used for the evaluation of gene stability (31). In each of the experimental differentiation procedures, the BMSCs group was defined as the calibrator sample, and the data of each differentiated lineage was combined with the BMSC group (30).

The resulting $\mathrm{Ct}$ values demonstrated that GAPDH exhibited the lowest mean $\mathrm{Ct}$ values, followed by ACTB and CYCA. ACTB was observed to exhibit the highest variation in RNA expression levels. The lowest level of RNA expression variation was observed in TBP, followed by CYCA. This revealed that the quantities of the reference genes varied markedly. The specificity of the $18 \mathrm{~s}$ rRNA designed primer easily amplifies nonspecific bands, and in vivo data has revealed that $18 \mathrm{~S}$ rRNA is unfit as a reference gene in neurons (31). A possible explanation for this may be that, when $18 \mathrm{~S}$ rRNA is used as a reference gene, it may be associated with an imbalance between mRNA and rRNA. Therefore, 18S rRNA was not included in the presents study.

GeNorm, NormFinder and BestKeeper software were used to rank the analyzed reference genes according to their expression stabilities. This rank order differed marginally between these tools, possibly due to the fact they are based on different mathematical models (32). All the software indicated that RPL13A exhibited the highest stability, and, ACTB was affirmed as the least stable gene by all three algorithms. This suggested that it was not possible to estimate the stability of a reference gene using the $\mathrm{Ct}$ value alone. These results are the same as those of a previous study. Bonefeld et al validated eight reference genes in rat hippocampal tissue neurons in vivo and also identified CYCA and RPL13A as the most stably expressed genes, and ACTB and 18S rRNA as the least stable genes (33). In a previous study investigating suitable reference genes for qPCR in the dentate gyrus following experimental febrile seizures, geNorm and Normfinder also indicated CYCA, RPL13A and TBP as the most stable genes, whereas $18 \mathrm{~S}$ rRNA and ACTB were found to be the least stably expressed genes (31).

The optimal number of reference genes for normalization is suggested by a $\mathrm{V}$ value below the cut-off of 0.15 in geNorm (17). The results of the present study indicated that the normalization factor requires two reference genes. As indicated in Fig. 6, the combination of RPL13A and HPRT were identified as the optimal pair for evaluating gene stability, however, according to NormFinder (Fig. 7), the results indicated that CYCA and PIPA were the optimal pair of reference genes. Based on the above results, the present study recommended that the pair of RPL13A and HPRT were optimal for the evaluation of gene stability, according to ranking of the genes from 1-12 for stability. According to Fig. 5, a pair-wise variation of 0.118 was observed following the addition of the second most stable gene.

In conclusion, the present study investigated the expression stabilities of 11 reference genes, which are commonly used for normalization in gene analysis, in three types of differentiated neuron cell from BMSCs and normal BMSCs. The results demonstrated that RPL13A, and the combination of CYCA and PPIA provided a reliable interpretation of the mRNA expression data in the experimental set-up, while ACTB appeared unsuitable as a reference gene. These results can be consulted for further qPCR investigations, and also present an appropriate strategy for the evaluation of candidate reference genes for any BMSC differentiation procedure.

\section{Acknowledgements}

This study was supported by the Project (GAP-43 gene modified bone marrow mesenchymal stem cells can differentiate into ganglion-like cells and promote nerve regeneration) supported by the National Natural Science Foundation of China (grant. no. 30973265).

\section{References}

1. Bustin SA, Benes V, Garson JA, et al: The MIQE guidelines: minimum information for publication of quantitative real-time PCR experiments. Clin Chem 55: 611-622, 2009.

2. Huggett J, Dheda K, Bustin S and Zumla A: Real-time RT-PCR normalisation; strategies and considerations. Genes Immun 6: 279-284, 2005.

3. Bustin SA and Nolan T: Pitfalls of quantitative real-time reverse-transcription polymerase chain reaction. J Biomol Tech 15: 155-166, 2004.

4. Zhong Q, Zhang Q, Wang Z, et al: Expression profiling and validation of potential reference genes during Paralichthys olivaceus embryogenesis. Mar Biotechnol (NY) 10: 310-318, 2008.

5. Bustin SA, Benes V, Nolan T and Pfaffl MW: Quantitative real-time RT-PCR-a perspective. J Mol Endocrinol 34: 597-601, 2005.

6. Dheda K, Huggett JF, Bustin SA, Johnson MA, Rook G and Zumla A: Validation of housekeeping genes for normalizing RNA expression in real-time PCR. Biotechniques 37: 112-114, 116, 118-119, 2004.

7. Radonic A, Thulke S, Mackay IM, Landt O, Siegert W and Nitsche A: Guideline to reference gene selection for quantitative real-time PCR. Biochem Biophys Res Commun 313: 856-862, 2004.

8. Suzuki T, Kobayashi K and Sasaki O: Real-time displacement measurement with a two-wavelength sinusoidal phase-modulating laser diode interferometer. Appl Opt 39: 2646-2652, 2000.

9. Cordoba EM, Die JV, González-Verdejo CI, Nadal S and Román B: Selection of reference genes in Hedysarum coronarium under various stresses and stages of development. Anal Biochem 409: 236-243, 2011.

10. Schmittgen TD and Zakrajsek BA: Effect of experimental treatment on housekeeping gene expression: validation by real-time, quantitative RT-PCR. J Biochem Biophys Methods 46: 69-81, 2000.

11. Santos AR and Duarte CB: Validation of internal control genes for expression studies: effects of the neurotrophin BDNF on hippocampal neurons. J Neurosci Res 86: 3684-3692, 2008. 
12. Sun C, Shao J, Su L, et al: Cholinergic neuron-like cells derived from bone marrow stromal cells induced by tricyclodecane-9-yl-xanthogenate promote functional recovery and neural protection after spinal cord injury. Cell Transplant 22: 961-975, 2013.

13. Woodbury D, Schwarz EJ, Prockop DJ and Black IB: Adult rat and human bone marrow stromal cells differentiate into neurons. J Neurosci Res 61: 364-370, 2000.

14. Sanchez-Ramos J, Song S, Cardozo-Pelaez F, et al: Adult bone marrow stromal cells differentiate into neural cells in vitro. Exp Neurol 164: 247-256, 2000.

15. Xu C, Su G and Mu D: Retinal cells of rat neonates inducing the bone marrow mesenchymal cells into RGC-like cells. Chin Ophthal Res 26: 174-178, 2008.

16. Wang C, Su G, Qi S, et al: Study of GAP-43 expression on differentiation of bone mesenchymal stem cells to neuron-like cells. Chin J Lab Diagn 12: 1206-1209, 2008.

17. Vandesompele J, De Preter K, Pattyn F, et al: Accurate normalization of real-time quantitative RT-PCR data by geometric averaging of multiple internal control genes. Genome Biol 3 RESEARCH0034, 2002.

18. Andersen CL, Jensen JL and Ørntoft TF: Normalization of real-time quantitative reverse transcription-PCR data: a model-based variance estimation approach to identify genes suited for normalization, applied to bladder and colon cancer data sets. Cancer Res 64: 5245-5250, 2004.

19. Pfaffl MW,Tichopad A,PrgometC and NeuviansTP:Determination of stable housekeeping genes, differentially regulated target genes and sample integrity: BestKeeper-excel-based tool using pair-wise correlations. Biotechnol Lett 26: 509-515, 2004

20. Long X, Olszewski M, Huang W and Kletzel M: Neural cell differentiation in vitro from adult human bone marrow mesenchymal stem cells. Stem Cells Dev 14: 65-69, 2005.

21. Feng X, Xiong Y, Qian H, Lei M, Xu D and Ren Z: Selection of reference genes for gene expression studies in porcine skeletal muscle using SYBR green qPCR. J Biotechnol 150: 288-293, 2010

22. Aramant RB, Seiler MJ and Ball SL: Successful cotransplantation of intact sheets of fetal retina with retinal pigment epithelium Invest Ophthalmol Vis Sci 40: 1557-1564, 1999.
23. Gouras P, Flood MT, Kjedbye H, Bilek MK and Eggers H: Transplantation of cultured human retinal epithelium to Bruch's membrane of the owl monkey's eye. Curr Eye Res 4: 253-265, 1985.

24. Gimble JM, Zvonic S, Floyd ZE, Kassem M and Nuttall ME: Playing with bone and fat. J Cell Biochem 98: 251-266, 2006.

25. Dezawa M, Kanno H, Hoshino M, et al: Specific induction of neuronal cells from bone marrow stromal cells and application for autologous transplantation. J Clin Invest 113: 1701-1710, 2004

26. Gregory CA, Prockop DJ and Spees JL: Non-hematopoietic bone marrow stem cells: molecular control of expansion and differentiation. Exp Cell Res 306: 330-335, 2005.

27. Gu S, Xing C, Han J, Tso MO and Hong J: Differentiation of rabbit bone marrow mesenchymal stemcells into corneal epithelial cells in vivo and ex vivo. Mol Vis 15: 99-107, 2009.

28. Liu C, Xin N, Zhai Y, et al: Reference gene selection for quantitative real-time RT-PCR normalization in the half-smooth tongue sole (cynoglossus semilaevis) at different developmental stages, in various tissue types and on exposure to chemicals. PLoS One 9: e91715, 2014.

29. McCurley AT and Callard GV: Characterization of housekeeping genes in zebrafish: male-female differences and effects of tissue type, developmental stage and chemical treatment. BMC Mol Biol 9: 102, 2008.

30. Farrokhi A, Eslaminejad MB, Nazarian H, Moradmand A, Samadian A and Akhlaghi A: Appropriate reference gene selection for real-time Pcr Data normalization during rat mesenchymal stem cell differentiation. Cell Mol Biol (Noisy-le-grand) 58 (Suppl): OL1660-1670, 2012.

31. Swijsen A, Nelissen K, Janssen D, Rigo JM and Hoogland G: Validation of reference genes for quantitative real-time PCR studies in the dentate gyrus after experimental febrile seizures. BMC Res Notes 5: 685, 2012.

32. Chang E, Shi S, Liu J, et al: Selection of reference genes for quantitative gene expression studies in Platycladus orientalis (Cupressaceae) using real-time PCR. PLoS One 7: e33278, 2012.

33. Bonefeld BE, Elfving B and Wegener G: Reference genes for normalization: a study of rat brain tissue. Synapse 62: 302-309, 2008. 